\title{
Application of Virtual Laboratory in Biology Teaching
}

\author{
Jialin Guo \\ Luohe Medical College, Luohe, Henan, 462000
}

Keywords: Virtual laboratory; biology teaching; teaching quality

\begin{abstract}
The virtual laboratory comprehensively utilizes various high-tech to build a virtual simulation learning environment, and provides a new teaching mode and teaching method for traditional teaching. This paper briefly introduces the connotation of virtual laboratory, domestic and foreign development, and the practical application in biology teaching, and further expounds the advantages and disadvantages in practical application, in order to explore how to use virtual laboratory to complete biological teaching tasks more efficiently, and provide a certain reference for improving the quality of teaching.
\end{abstract}

\section{Introduction}

Biology is a discipline with a wide variety of theoretical knowledge and strict practice. Traditional daily teaching is often restricted by factors such as teacher strength, experimental equipment, teaching expenses, and biosafety. As a result, students' understanding of theoretical knowledge is not thorough, and experimental hands-on operation is lacking, which greatly affects teaching quality and teaching progress. In recent years, with the continuous development of computer network technology and multimedia technology, virtual labs are introduced into biology teaching, virtual auxiliary teaching is used to supplement regular teaching, and human-computer interaction and interaction have become a new direction of current teaching reform and development. The solution to the relative shortage of teaching resources is uneven, helping students to better grasp the knowledge points and improve practical skills.

\section{The Concept of Virtual Laboratory}

In 1989, Professor William Wolf of the University of Virginia first proposed the concept of virtual laboratory, and detailed an open platform that integrates various tool technologies and allows users to freely share data and conduct experiments by breaking through time and space constraints. With the continuous development of Internet and multimedia technology, mathematical modeling and simulation technology, virtual laboratories can realize three-dimensional simulation of real life processes, experimental processes, and physical and chemical phenomena on the basis of specific numerical models. The signals input into the virtual scene through the keyboard and mouse are calculated by software, and the specific operation results and experimental phenomena are visually outputted, and the entire process of the experimental system is simulated and reproduced.

\section{Overview of Domestic and Foreign Development of Virtual Laboratories}

The research and construction of virtual laboratories in foreign countries started early and developed rapidly. In 1985, the National Library of Medicine conducted a digital study of human anatomical images. Each of the male corpses and a female corpse was scanned by CT and MRI. The data was reconstructed in three dimensions to establish a "virtual human body" system, and further virtual anatomy was carried out. Computer-assisted instruction in the disciplines of virtual radiology and virtual endoscopy. In 1995, a popular "virtual frog anatomy experiment" on the Internet, the experimenter used a virtual operation scalpel to stratify the frog layer, which can clearly and intuitively observe the internal musculoskeletal and other organizational structures. Up to now, many foreign universities have developed their own virtual learning systems, such as the 
digital anatomist system developed by the University of Washington, USA, the nuclear medicine virtual simulation system developed by Rensselaer Polytechnic Institute, developed by the University of Hamburg, Germany. The Voxel-Man system, etc., users can effectively grasp theoretical knowledge and experimental techniques by using virtual experiment systems. At present, the most famous bio-virtual laboratories in the world are: Genetically modified laboratories, bacterial identification laboratories, heart disease laboratories, immunology laboratories, neurophysiological laboratories, etc. developed by Howard Hughes Medical Research Institute, Nobel Foundation The virtual biochemical laboratory, which was established, provides an important reference for the research and application of bio-virtual laboratories, and provides an important experimental base for the cultivation of biomedical talents.

Domestic virtual laboratories started late, but they have developed rapidly in recent years. Due to the characteristics of virtual simulation technology, it is widely used in science and engineering, especially in military, construction, machinery, electronics, biomedicine and other fields. At present, universities, hospitals and research institutes such as Tsinghua University, Peking University, China Agricultural University, and West China Clinical Medical College of Sichuan University have successively carried out research on virtual experimental technology, and started to develop virtual biology laboratory, virtual medical laboratory, and remote. Various virtual experimental systems, such as teaching systems, involve teaching, scientific research, and commercial fields. The functions are also developed from simple information simulation to a network cooperation and sharing platform based on virtual resources, scenarios, and models. At the same time, some virtual experimental teaching software has appeared in the education market, such as the biological function experiment system developed by Chengdu Taimeng Technology Co., Ltd., the biological signal acquisition and analysis system, and the virtual anatomy laboratory system developed by Beijing Weituo Qichuang Information Technology Co., Ltd. . According to the "Several Opinions of the Ministry of Education on Comprehensively Improving the Quality of Higher Education" and the "Development Plan for the Ten Years of Education Informatization (2011-2020)", the country has launched the national virtual simulation experiment teaching center construction strategy in 2013. The construction of virtual laboratories in China is ushering in new development opportunities.

\section{The Specific Application of Virtual Laboratory in Biology Teaching}

The virtual laboratory teaches by constructing a realistic three-dimensional environment and applies it to traditional biology teaching, which will have a profound impact on the biological education mode, teaching methods and teaching methods. At present, virtual laboratories play an important role in the following aspects.

The experimental teaching process is an important link between theoretical knowledge and practical operation, and it is also the most important link for cultivating and improving students' innovative ability. The virtual laboratory uses virtual simulation technology to simulate the actual biological experiment process and phenomena, and visually reproduce the corresponding experimental process. In this vivid and realistic experimental environment, students can complete a series of steps including experimental design, experimental operation and analysis of experimental results. Experiments involving relatively expensive and dangerous consumables involving high-end instruments and equipment, and long-time experimental operations such as biochemistry and molecular biology experiments, microbiology experiments, bioengineering techniques, etc.

Learning, using virtual biology laboratories, can effectively solve the difficulties that are difficult to overcome in the above traditional experimental teaching process, improve the experimental teaching mode, and further improve the teaching quality and students' innovative ability.

The traditional anatomy teaching is mainly based on classroom face-to-face teaching, assisting with teaching tools such as pictures, specimens, models, etc., and with practical operations such as autopsy to deepen students' understanding of organizational structure. In recent years, with the continuous shortage of human body specimen resources for teaching, the quality of human anatomy teaching has been greatly restricted. The virtual anatomy teaching combines the frozen section technology and the three-dimensional image processing technology, and the section slice 
information is processed by the virtual simulation software to form a digital model of the physiological structure having a three-dimensional effect, which can be arbitrarily rotated, cut, and can be performed on a certain part. More in-depth anatomy. Compared to traditional teaching tools, it is

It has incomparable advantages in image clarity, texture and operability. In addition, it has the advantages of no pollution, no loss, and scalability, which makes it have broad application prospects in anatomy teaching.

The process of biological development is relatively abstract and complex, with many knowledge points and large time spans. It is often difficult for students to fully understand and master in time. The virtual developmental biology teaching system can completely, clearly and systematically restore cell growth cycle and embryo in a short time. Through the whole process of development, the long and boring teaching process is improved into a simple and vivid experimental experience, which enables students to fully understand the life processes such as cell division, embryogenesis and early development, which not only enhances the teaching interest, but also simplifies the knowledge system. Deepen students' understanding of knowledge points.

In the actual teaching process, the traditional tissue slicing is often not easily applied due to the large number, the difficulty of storage, the damage and the inconvenience of searching. The virtual slicing system converts tissue slices into digitized image text information, and collects tissue slice information represented by typical morphology structures, stores them according to different disciplines and chapters, and establishes a corresponding virtual slicing system. The virtual slicing system has the advantages of convenient searching, no loss, easy storage, and scalability, and to a certain extent, it can promote cooperation among various laboratories to realize resource sharing.

Bioinformatics is an emerging interdisciplinary subject that uses computer resources to develop various software to collect, organize, store, publish, extract, process, analyze and research biological information. It aims to gradually understand the origin, evolution and inheritance of life. And the nature of development. The Virtual Bioinformatics Lab uses a variety of network technologies and network tools to provide researchers with a common resource sharing service platform that is independent of time and geography. For example, NCBI of the National Library of Medicine provides a comprehensive digital information service, which is widely used by researchers all over the world. At present, the more important bioinformatics virtual experiments include electronic Northern, systematic analysis of gene expression, digital differential display, electronic cloning, electronic PCR, and electron in situ hybridization. Users can directly obtain the obtained gene or mRNA sequence in a public database. Comparison, hybridization, analysis, etc., automatically generate test results, convenient and fast.

\section{Application Advantages of Virtual Laboratory in Biology Teaching}

The virtual laboratory has the advantages of high efficiency, easy operation, openness, flexibility, powerful function, high cost performance, and free definition of users. It can make up for the shortcomings of traditional teaching, improve teaching efficiency and teaching quality, and thus is widely respected by universities.

The virtual laboratory integrates information such as images, texts, sounds, and animations to generate a digital environment that is highly similar to a real range of visual, auditory, and tactile aspects, realizing and abstracting abstract concepts and principles. , keyboards, etc. submit different operational instructions and accept feedback information to complete human-computer interaction, so that students have an immersive experience and experience. This kind of immersion and interactivity makes students truly become participants in the experiment. They are in a state of excitement and thinking throughout the whole process, which fully stimulates students' interest in learning and greatly enhances the enthusiasm and initiative of learning.

The virtual laboratory is based on the development of the network, so that the teaching process is no longer limited by time, geography and experiment times. Teachers and students can participate at any time and place, exchange and interact, and obtain teaching resources. In the actual teaching, some biological experiments involve a long experimental period. For example, the establishment of 
knockout mice requires that the mice be crossed for several generations to obtain homozygous mice. In the virtual experiment, this long development process can be It is vividly displayed in front of students in a short time. In addition, for some experiments that may involve expensive equipment consumables and specific experimental sites, the use of virtual laboratories can significantly reduce laboratory construction, equipment consumables purchase, energy consumption and maintenance costs, and reduce the need for real experimental equipment. And the dependence of consumables, saving a lot of experimental funding.

In real biological experiments, it is inevitable to be exposed to some toxic, harmful, flammable, explosive, radioactive reagents, which will cause irreversible damage to the experimenter and the environment, and also limit the development of some experiments. In addition, due to insufficient student experience, operational errors can have unpredictable and dangerous consequences. If in the virtual experiment operation, the danger signal prompt and the dangerous scene forecast are set in the key position or the dangerous step, it is helpful to deepen the student's impression and cultivate good experimental habits, so as to avoid rushing in the actual operation and avoiding the safety and hidden danger. In addition, the use of virtual laboratories can also reduce the environmental pollution and personal safety caused by the discharge of a large number of discarded reagents in real-life experiments.

At present, all major universities have their own experimental teaching centers. They have both individuality and commonality. They are relatively independent and interrelated. They can use the advantages of network sharing in virtual laboratories to achieve interdisciplinary, interdisciplinary and interdisciplinary Sharing resources, strengthen cooperation and exchanges between each other, and promote optimal allocation of resources.

\section{Problems in Virtual Laboratory in Biology Teaching}

Although virtual labs have many advantages, they also have some drawbacks. In the actual teaching, it can not completely replace the real laboratory, nor can it blindly completely virtualize the experiment in the textbook and abandon the reality experiment. At present, the virtual experiment has the following problems in practical application.

(1) Virtual experiments cannot fully reflect real-life experiments. The virtual experiment operation is a kind of virtual experiment in the simulated environment. Some unknown factors in the actual experiment process (different batches of experimental materials, different manufacturers' experimental equipment, human error) may cause errors in the experimental results, which cannot be completely Reflecting the reality process, it cannot fully express unexpected situations in the real environment. Therefore, teachers should treat virtual laboratories rationally, and cannot entrust virtual teaching in full. Instead, they should choose appropriate virtual experimental content to assist daily teaching.

(2) Due to technical research and development restrictions, the virtual experiment mode is still relatively simple and closed at this stage. At present, the biological virtual experiment system purchased or independently researched by major universities is still a relatively basic level. The experimental content, steps and phenomena are preset. Users can only operate according to the set procedures. This mode limits the teaching content and teachers. The teaching expression is not conducive to the interaction between teachers and students to a certain extent, but also limits the way students think, which is not conducive to the creativity and individuality of students. Therefore, the development of virtual experiment system needs to be further immersed at a higher level. It is more sexual and develops in a direction that can flexibly adapt to different teaching environments.

(3) Lack of teamwork and communication in virtual experiments. In the actual experimental operation, each member of the experimental group usually needs to cooperate with each other to successfully complete the experimental task. During the period, the students' teamwork awareness is gradually cultivated, and communication and communication between each other is enhanced, which is beneficial to the wider use of each student. Talent, improve personal comprehensiveness quality. The virtual experiment can be completed in the virtual environment only by the students themselves, and lacks the opportunity to cultivate students' cooperation, communication and 
communication skills. Therefore, virtual laboratories should complement and promote each other with real-life experiments to be more conducive to the cultivation of innovative talents.

\section{Conclusion}

As a new form of teaching, virtual experiment teaching has the advantage that traditional teaching methods can't match. It can provide students with an intuitive and convenient operating environment, which is conducive to students' understanding of theoretical knowledge, stimulating learning motivation and improving teaching quality. As a supplement and extension of the traditional biology teaching mode, virtual experiment teaching has a fresh vitality. Although there are currently some shortcomings, we believe that with the continuous updating of science and technology and the further improvement of teaching methods, virtual laboratories are teaching biology. There will be good development prospects in the field.

\section{References}

[1] Tang Mingxiang, Yang Gongming, Li Kaixiong. Food Science Virtual Laboratory based on virtual reality technology [J]. Journal of Shihezi University (Natural Science Edition), 2005, 23(2): 158-162.

[2] Zhong Shizhen. The scientific significance and application prospect of digital virtual human body [J]. Journal of First Military Medical University, 2003 23(3): 193-195.

[3] Jiang Haiping, Feng Hong. The impact of the development of virtual reality technology on biological experiments and teaching [J]. Genetics, 2007, 29(12): 1529-1532.

[4] Pu Dan, Zhou Zhou, Ren An Jie, et al. Preliminary study on the construction experience of multi-level comprehensive virtual simulation experiment teaching center [J]. Experimental Technology and Management, 2014, 31(3): 5-8.

[5] Xie Lifang, Lin Honghui. The role of virtual laboratory in undergraduate biological experiment teaching [J]. Experimental Technology and Management, 2014, 31(9): 114-116.

[6] Li Yun, Luo Gang, Ling Guanhua, et al. The application of virtual reality technology in medical education [J]. Laboratory Medicine and Clinical Medicine, 2014, 11(17): 2485-2487.

[7] Wang Xinhai, Li Weiguang, Xu Ying. Application of virtual reality technology in chemistry experiment teaching [J]. Guangzhou Chemical Industry, 2014, 42(13): 214-215 